Compared to C-GCA, patients with large vessel involvement (LV-GCA and LV-CGCA) were younger and more frequently women, and the difference was further significant for patients with isolated LV-GCA. Patients with isolated LV-GCA had also the longer duration of symptoms at GCA diagnosis [LV-GCA 20(16-82) vs LV-C-GCA 12(4-16) vs C-GCA 4(3-12) weeks; $p<0.001]$. Systemic symptoms, as fever and fatigue, were associated with large vessel involvement, both in LV-GCA and LV-C-GCA groups. Polymyalgia rheumatica was equally reported in all three cohorts of patients and no significant differences were found in inflammatory markers levels according to vessel involvement.

In patients with large vessels involvement (LV-GCA and LV-C-GCA) thoracic aorta, subclavian arteries and abdominal aorta were the most frequently involved arteries.

Conclusion: GCA is not a single entity but includes several patterns of disease. Female gender, younger age and systemic symptoms are associated with large vessel involvement, regardless the presence or absence of cranial symptoms. The different clinical manifestations of large vessel GCA lead to a longer time to diagnosis if compared to C-GCA. For these reasons, in patients with the aforementioned characteristics, a large vessel involvement should be considered in order to reduce the time to diagnosis.

REFERENCES:

[1] De Boysson H, et al. Clin Exp Rheumatol 2019.

[2] Prieto-González S, et al. Ann Rheum Dis 2012.

[3] Brack A, et al. Arthritis Rheum 1999

[4] Muratore F, et al. Rheumatol 2015.

\begin{tabular}{|c|c|c|c|c|c|}
\hline & $\begin{array}{c}\text { GCA } \\
(n: 100)\end{array}$ & $\begin{array}{l}\text { C-GCA } \\
\text { (n: 61) }\end{array}$ & $\begin{array}{c}\text { LV-C-GCA } \\
(\mathrm{n}: 23)\end{array}$ & $\begin{array}{c}\text { LV-GCA } \\
\text { (n: 16) }\end{array}$ & $\mathrm{p}$ \\
\hline Age: median (IQR) & $76(67-79)$ & $75(71-80)$ & $74(69-77)$ & $63(59-72)$ & 0.001 \\
\hline \multirow[t]{2}{*}{ Female/ Male } & $68(68 \%) /$ & $\begin{array}{l}38(62 \%) / \\
23(38 \%)\end{array}$ & $17(74 \%) /$ & $13(81 \%) /$ & 0.276 \\
\hline & $32(32 \%)$ & & $6(26 \%)$ & $3(19 \%)$ & \\
\hline $\begin{array}{l}\text { Time between symptom onset and } \\
\text { diagnosis (weeks) }\end{array}$ & $8(4-20)$ & $4(3-12)$ & $12(4-16)$ & $20(16-82)$ & $<0.001$ \\
\hline Cranial symptoms (overall) & $84(84 \%)$ & $61(100 \%)$ & $23(100 \%)$ & $0(0 \%)$ & $<0.001$ \\
\hline New temporal headache & $77(77 \%)$ & $58(95 \%)$ & $19(83 \%)$ & $0(0 \%)$ & $<0.001$ \\
\hline Visual symptoms & $39(39 \%)$ & $34(56 \%)$ & $5(22 \%)$ & $0(0 \%)$ & $<0.001$ \\
\hline Jaw or tongue claudication & $35(35 \%)$ & $27(44 \%)$ & $8(35 \%)$ & $0(0 \%)$ & 0.004 \\
\hline Fever & $48(48 \%)$ & $23(38 \%)$ & $14(61 \%)$ & $11(69 \%)$ & 0.032 \\
\hline Fatigue & $75(75 \%)$ & $40(66 \%)$ & $21(91 \%)$ & $14(88 \%)$ & 0.023 \\
\hline Weight loss & $52(52 \%)$ & $28(46 \%)$ & $13(57 \%)$ & $11(69 \%)$ & 0.235 \\
\hline Polymyalgia rheumatica & $43(43 \%)$ & $27(44 \%)$ & $13(57 \%)$ & $3(19 \%)$ & 0.061 \\
\hline Arm or leg claudication & $5(5 \%)$ & $0(0 \%)$ & $4(17 \%)$ & $1(6 \%)$ & 0.005 \\
\hline CRP (C-reactive protein) & $83(45-127)$ & 77 (39-115) & $89(50-134)$ & ) $95(20-124)$ & 0.461 \\
\hline $\begin{array}{l}\text { ESR (erythrocyte sedimentation } \\
\text { rate) }\end{array}$ & $72(47-96)$ & $70(46-88)$ & $75(28-105)$ & $66(44-91)$ & 0.711 \\
\hline Cranial arteries & \%) & $0 \%)$ & 23 & 0 & $<0.001$ \\
\hline Carotid arteries & $19(19 \%)$ & $0(0 \%)$ & $12(52 \%)$ & $7(44 \%)$ & $<0.001$ \\
\hline Subclavian and upper limb arteries & s $21(21 \%)$ & $0(0 \%)$ & 10 (43\%) & $11(69 \%)$ & $<0.001$ \\
\hline Thoracic aorta & $29(29 \%)$ & $0(0 \%)$ & $15(65 \%)$ & $14(88 \%)$ & $<0.001$ \\
\hline Abdominal aorta & $21(21 \%)$ & $0(0 \%)$ & $10(43 \%)$ & $11(69 \%)$ & $<0.001$ \\
\hline Iliac and inferior limb arteries & $10(10 \%)$ & $0(0 \%)$ & $6(26 \%)$ & $4(25 \%)$ & $<0.001$ \\
\hline
\end{tabular}

Disclosure of Interests: None declared

DOI: 10.1136/annrheumdis-2021-eular.510

GIANT CELL ARTERITIS TREATMENT PATTERNS AND RATES OF SERIOUS INFECTIONS

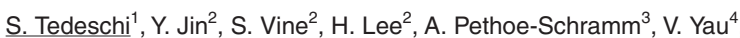
S. Kim ${ }^{1,2} .{ }^{1}$ Brigham and Women's Hospital, Rheumatology, Inflammation and Immunity, Boston, United States of America; ${ }^{2}$ Brigham and Women's Hospital, Pharmacoepidemiology and Pharmacoeconomics, Boston, United States of America; ${ }^{3}$ F. Hoffmann-La Roche Ltd., Global Medical Affairs - Immunology, Basel, Switzerland; ${ }^{4}$ Genentech, Personalized Healthcare Data Science, South San Francisco, United States of America

Background: Giant cell arteritis (GCA) afflicts older adults, who may have ageand comorbidity-related risks for infection, and is treated with glucocorticoids and other immunosuppressants that might increase the risk of serious infections Objectives: To examine GCA treatment patterns and rates of serious infections in two real-world cohorts in the U.S.

Methods: Using claims data from two U.S. health insurance databases, Medicare (public, 2007-2017) and MarketScan (commercial, 2015-2019), we selected a cohort of patients with GCA based on a validated claims-based algorithm requiring $\geq 2$ diagnosis codes for GCA plus dispensing of high-dose oral glucocorticoid (PPV 84.8\%) [1]. The Medicare cohort included Medicare enrollees aged $\geq 65$ years with GCA who were treated at a large Massachusetts-based healthcare system; the MarketScan cohort included a nationwide sample of GCA patients aged $\geq 50$ years. GCA index date was the date of $1^{\text {st }}$ glucocorticoid prescription. We assessed baseline comorbidities in the year prior to index date. Immunosuppressants and prophylactic antibiotics dispensed within 30 days of index date were recorded. Influenza vaccine administration in the year after index date was identified. We calculated the incidence rate of serious infections, defined as infections requiring hospitalization, after index date through end of cohort follow-up (12/31/17 in Medicare; 12/31/2019 in MarketScan), disenrollment, or death.

Results: The Medicare cohort included 734 patients, 28\% male, mean age 77.1 (SD 7.4); the MarketScan cohort included 1022 patients, $30 \%$ male, mean age 68.4 (SD 10.9). The most common comorbidity was hypertension (80\% Medicare; $72 \%$ MarketScan) followed by hyperlipidemia, cancer, coronary artery disease, and diabetes (Table 1). High-dose prednisone was the most common initial treatment; a small percentage received tocilizumab within 30 days of index date. Prophylaxis against pneumocystis pneumonia was similar in Medicare (13\%) and MarketScan cohorts (10\%). Influenza vaccination was more common in the Medicare (56\%) than MarketScan (22\%) cohort in the year after GCA index date. During the mean follow-up time of 2.6 (SD 2.5) years in Medicare and 1.5 (SD 0.9) years in MarketScan, $27.9 \%$ of the Medicare and $7.2 \%$ of MarketScan patients developed serious infections: incidence rate per 100 person-years = 10.7 (95\% Cl 9.3, 12.2) in Medicare and $6.3(95 \% \mathrm{Cl} 5.0,7.9)$ in MarketScan. Conclusion: In these 2 real-world GCA cohorts in the US, over 25\% of Medicare and $7 \%$ of MarketScan patients developed serious infection during follow-up. The incidence rate of serious infection was similar to ANCA-associated vasculitis [2] Use of prophylactic antibiotics and influenza vaccination was suboptimal among GCA patients.

REFERENCES:

[1] Lee H, et al. ACR Open Rheum 2020 (in press).

[2] Rathmann J, et al. Rheumatology 2020; doi: 10.1093/rheumatology/keaa699.

Table 1. Comorbidities, medication use, and infection rates in GCA cohorts

\begin{tabular}{lcc}
\hline & $\begin{array}{c}\text { Medicare cohort } \\
(\mathbf{n}=734)\end{array}$ & $\begin{array}{c}\text { MarketScan } \\
\text { cohort (n=1022) }\end{array}$ \\
\hline Baseline comorbidities & & \\
Hypertension & $80 \%$ & $72 \%$ \\
Hyperlipidemia & $71 \%$ & $61 \%$ \\
Cancer & $38 \%$ & $24 \%$ \\
Coronary artery disease & $34 \%$ & $25 \%$ \\
Diabetes & $34 \%$ & $31 \%$ \\
No. rheumatology visits, mean (SD) & $1.4(2.2)$ & $0.8(1.7)$ \\
Medications within 30 days of index date & $87 \%$ & $91 \%$ \\
Prednisone maximum dose $\geq 60$ mg daily & $<2 \%$ & $5 \%$ \\
Tocilizumab IV or SC & $3 \%$ & $4 \%$ \\
Methotrexate & $13 \%$ & $10 \%$ \\
Bactrim, atovaquone, or dapsone & & $22 \%$ \\
Vaccines in the year after index date & $56 \%$ & $6.3(5.0,7.9)$ \\
Influenza vaccine & $10.7(9.3,12.2)$ & \\
Serious infection incidence rate (95\% Cl) per 100 & & \\
$\quad$ person-years & & \\
\hline
\end{tabular}

Disclosure of Interests: Sara Tedeschi: None declared, Yinzhu Jin: None declared, Seanna Vine: None declared, Hemin Lee: None declared, Attila Pethoe-Schramm Employee of: F. Hoffmann-La Roche, Vincent Yau Employee of: F. Hoffmann-La Roche/Genentech, Seoyoung Kim Grant/research support from: Roche, Pfizer, AbbVie and Bristol-Myers Squibb

DOI: 10.1136/annrheumdis-2021-eular.561

\section{POS0799 THE EFFICACY AND SAFETY OF TOCILIZUMAB IN PATIENTS WITH GIANT CELL ARTERITIS: A SYSTEMATIC REVIEW AND META-ANALYSIS}

M. Koster ${ }^{1}$, K. J. Warrington', J. Han², S. Mohan ${ }^{3} .{ }^{1}$ Mayo Clinic, Division of Rheumatology, Rochester, United States of America; ${ }^{2}$ Genentech, Inc., Biometrics, South San Francisco, United States of America; ${ }^{3}$ Genentech, Inc., Immunology, South San Francisco, United States of America

Background: Tocilizumab (TCZ) has been proven to be safe and effective for the treatment of giant cell arteritis (GCA) in 2 randomized controlled trials; however, data from additional types of studies provide valuable information related to the treatment of GCA with TCZ.

Objectives: To review and analyze efficacy and safety data for TCZ in GCA based on peer-reviewed publications to date.

Methods: A systematic literature review was conducted according to the PRISMA guidelines. Publications were retrieved from the MEDLINE, Embase, Cochrane, Scopus and Web of Science databases. Publications of clinical trials and retrospective or prospective observational studies (April 11, 2005-October 8,2019 ) including patients with GCA (classified based on ACR criteria and/or positive biopsy vs imaging) treated with TCZ and reporting a measure of efficacy were eligible for inclusion. Extracted data included year of publication, year(s) when the study was conducted, number of patients with GCA, method of GCA diagnosis, age and sex, TCZ treatment details (dose, route of administration, frequency, duration), clinical outcome (remission, relapse), serious adverse events 